\title{
Characteristics and outcomes of patients who underwent bedside surgical tracheostomy performed in the intensive care unit: a retrospective study
}

\author{
Gabriella Jacob, BSc $\cdot$ Marnie Jakab, BSc $\cdot$ Jessica Ray, BSc
}

Received: 12 September 2017/Revised: 5 November 2017/ Accepted: 6 November 2017/Published online: 17 November 2017

(C) Canadian Anesthesiologists' Society 2017

\section{To the Editor,}

Among critically ill patients, progression to tracheostomy for those requiring extended ventilatory support has become routine. Bedside tracheostomy has been proposed as preferable to tracheostomy in the operating room (OR) due to lower costs, reduced wait times, and avoidance of transport of critically ill patients to the OR. ${ }^{1-3}$ We conducted a retrospective chart review of mechanically ventilated patients in the Mount Sinai Hospital intensive care unit (ICU) who underwent nonurgent bedside surgical tracheostomy (BST) to evaluate complications and outcomes.

Between February 2009 and April 2013, there were 97 patients who required BST in the ICU. Their mean (SD) age was 59 (17) yr, Acute Physiology and Chronic Health Evaluation II score was 27 (8), and 53\% were male. Most of the patients were admitted for respiratory failure $(n=60,62 \%)$, primarily from another acute care facility $(n=44,45 \%)$ or from home $(n=39,40 \%)$. Tracheostomy was performed on median [interquartile (IQR)] day 15 [8-21] after intubation. Most of the patients

G. Jacob, BSc ( $\square)$

Faculty of Medicine, University of Toronto, Toronto, ON, Canada

e-mail: gabi.jacob@mail.utoronto.ca

G. Jacob, BSc · M. Jakab, BSc · J. Ray, BSc

Department of Medicine \& Interdepartmental Division of

Critical Care, Mount Sinai Hospital, Toronto, ON, Canada

M. Jakab, BSc

School of Medicine, Queen's University, Kingston, ON, Canada

J. Ray, BSc

Department of Medical Biophysics, University of Toronto,

Toronto, ON, Canada underwent tracheostomy for prolonged weaning ( $n=87$, $90 \%)$.

Most patients were given some combination of propofol, fentanyl, midazolam, rocuronium, and phenylephrine, with the median [IQR] duration of the tracheostomy procedure being 45 [35-60] min. Complications during the procedure occurred in eight patients and included desaturation, excessive bleeding, and hypotension (Table). Minor complications (e.g., bleeding) were relatively common after the procedure, whereas severe complications (e.g., accidental decannulation, barotrauma, tracheomalacia, subglottic stenosis) were rare (Table).

Three days prior to tracheostomy, $23 \%(n=22 / 96)$ and $27 \%(n=26 / 96)$ of patients were receiving sedative or opioid infusions, respectively. These percentages fell to $10 \%(n=9 / 92)$ and $18 \%(n=17 / 92)$, respectively, on day 3 , likely reflecting increased adaptive patient comfort. Most patients were successfully weaned from the ventilator $(n=63,65 \%)$, with a median [IQR] duration of mechanical ventilation of 21 [14-31] days. Hospital survivors $(n=61,63 \%)$ had a median [IQR] hospital length of stay of 60 [35-102] days. A large proportion of patients were discharged with a tracheostomy $(n=38$, $39 \%)$ or died with a tracheostomy in place $(n=34,35 \%)$. In all, 25 (26\%) patients were decannulated in hospital, and seven patients $(7 \%)$ were discharged directly home.

Although BST is common, most centres utilize a percutaneous dilational technique (PDT). ${ }^{3}$ The trend toward PDT at the bedside is related to the relative ease of the procedure and a shorter procedure time. ${ }^{3}$ Following several episodes of damage to the flexible bronchoscope during PDT, our centre opted for BST performed by the otolaryngology service. Most studies have reported similar rates of serious perioperative complications with bedside PDT and BST. ${ }^{3,4}$ The overall perioperative complication 
Table Intraoperative and postoperative early (within $48 \mathrm{hr}$ ) and late (after $48 \mathrm{hr}$ ) complications associated with open bedside tracheostomy

\begin{tabular}{|c|c|}
\hline & $n(\%)$ \\
\hline \multicolumn{2}{|l|}{ Intraoperative complications } \\
\hline Desaturation & $4(4 \%)$ \\
\hline Excessive bleeding & $3(3 \%)$ \\
\hline Hypotension & $1(1 \%)$ \\
\hline \multicolumn{2}{|l|}{ Postoperative early complications } \\
\hline Bleeding at tracheostomy site & $22(23 \%)$ \\
\hline Mucous plug & $3(3 \%)$ \\
\hline Accidental decannulation & $2(2 \%)$ \\
\hline Apneic period & $2(2 \%)$ \\
\hline Subcutaneous emphysema & $1(1 \%)$ \\
\hline Pneumothorax & $1(1 \%)$ \\
\hline Bradycardia & $0(0 \%)$ \\
\hline \multicolumn{2}{|l|}{ Postoperative late complications } \\
\hline Tracheostomy site erythema/necrosis & $26(27 \%)$ \\
\hline Mucous plug & $25(26 \%)$ \\
\hline Bleeding at tracheostomy site & $17(18 \%)$ \\
\hline Accidental decannulation & $10(10 \%)$ \\
\hline Barotrauma* & $5(5 \%)$ \\
\hline Infection of stoma & $2(2 \%)$ \\
\hline Tracheomalacia & $2(2 \%)$ \\
\hline Subglottic stenosis & $2(2 \%)$ \\
\hline Bradycardia & $1(1 \%)$ \\
\hline Other** & $5(5 \%)$ \\
\hline
\end{tabular}

*Barotrauma included three patients with pneumothorax, one with pneumomediastinum, and one with subcutaneous emphysema. **Other $=$ tracheal suction trauma, transient apnea, cardiac arrest, tracheostomy malposition, pleural effusion.

rate in our study was higher than that reported in a prior meta-analysis $(8 \% \text { vs } 3 \%)^{4}$ of 21 non-randomized trials. Those studies, however, rarely reported minor complications, such as transient desaturation, ${ }^{4}$ which in our study accounted for $50 \%$ of perioperative complications. While examining postoperative surgical notes and intraoperative nursing notes, we identified complications that may have been overlooked in other studies. Regarding postoperative complications, metaanalyses comparing BST and PDT have been inconsistent. $^{3,4}$ As a result, the consensus is for practitioners to choose the technique with which they have the most experience.

Compared with a large retrospective study of 1,445 patients in North Carolina, ${ }^{5}$ our study had a similar number of patients discharged home or who died in hospital. We had fewer patients discharged to long-term care and rehabilitation hospitals, however, with more discharged to another acute-care facility, which limited determination of the final discharge destination.

Overall, our data and the current literature suggest that BST in critically ill, mechanically ventilated patients is associated with a relatively low incidence of major perioperative complications. These patients, however, experience significant in-hospital mortality and low decannulation rates prior to discharge.

Acknowledgements We thank the following individuals for assistance in data collection, data analysis, and review of the manuscript: Brittany Lindsay, Alan Kraguljac, Dr. Anjuli Little, and Dr. Sangeeta Mehta.

\section{References}

1. Yoo DB, Schiff BA, Martz, $S$, et al. Open bedside tracheotomy: impact on patient care and patient safety. Laryngoscope 2011; 121 : 515-20.

2. Wang SJ, Sercarz JA, Blackwell KE, Aghamohammadi M, Wang $D B$. Open bedside tracheotomy in the intensive care unit. Laryngoscope 1999; 109: 891-3.

3. Oliver ER, Gist A, Gillespie B. Percutaneous versus surgical tracheotomy: an updated meta-analysis. Laryngoscope 2007; 117: 1570-5.

4. Durbin $C G J r$. Early complications of tracheostomy. Respir Care 2005; 50: 511-5.

5. Cox CE, Carson SS, Holmes GM, Howard C, Carey TS. Increase in tracheostomy for prolonged mechanical ventilation in North Carolina, 1993-2002. Crit Care Med 2004; 32: 2219-26. 